\title{
Pneumolysin: stimulating protection
}

Pneumolysin, a key Streptococcus pneumoniae virulence factor, is a cholesterol-dependent cytolysin that creates pores in cholesterol-containing membranes, thus causing host cell lysis. Pneumolysin has been proposed as a potential pneumococcal vaccine candidate, but although its cytolytic effects are well understood, less is known about the interactions between this potent toxin and the host immune system. Now, a new paper in PLoS Pathogens provides an insight into the immunomodulatory effects of this toxin.

In earlier work, conflicting results had been obtained about whether Toll-like receptor 4 (TLR4) has a role in host resistance to pneumo-

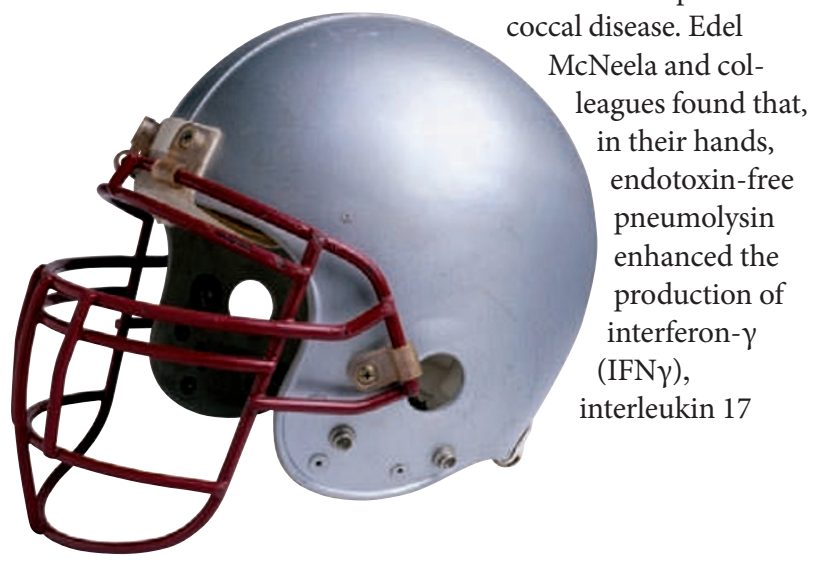

(IL-17) and other pro-inflammatory cytokines by stimulated splenocytes in a TLR4-independent manner. A pneumolysin-deficient strain of $S$. pneumoniae showed reduced virulence compared with wild type in a mouse model of pneumococcal infection, and this was correlated with reduced production of IFN $\gamma$ and IL-17, mainly from natural killer cells and $\gamma \delta$ T cells. So, pneumolysin promotes the production of IFN $\gamma$ and IL-17 in vitro and in vivo, and does not require TLR4 to do so.

Previous work had shown that $\mathrm{IL}-1 \beta$ is also required for resistance to pneumococcal infection. As IL- $1 \beta$ secretion requires activation of the IL- $1 \beta$ zymogen by caspase 1 , and this in turn requires assembly of the NLRP3 (NOD-, LRR- and pyrin domain-containing 3) inflammasome, McNeela et al. went on to look at the involvement of this multiprotein inflammasome complex in resistance to pneumococcal infection. They found that the ability of pneumolysin to promote IL-1 $\beta$ secretion from dendritic cells (DCs) was lower in $\mathrm{Nlrp}^{3^{-1-}}$ mice than in wild-type mice, indicating that pneumolysin promotes secretion of IL- $1 \beta$ via the NLRP3 inflammasome. Live S. pneumoniae also induced robust pneumolysin- and NLRP3 inflammasome-dependent IL- $1 \beta$ secretion by DCs. Finally, the authors found that clearance of $S$. pneumoniae infection was less efficient in $\mathrm{Nlrp}^{-1-}$ mice than in wild-type mice.

These results indicate that the NLRP3 inflammasome has a key role in protection against $S$. pneumoniae infection. This is the first demonstration of the involvement of the NLRP3 inflammasome in protection against a Gram-positive pathogen, and supports further investigation of pneumolysin as a potential pneumococcal vaccine candidate.

Sheilagh Molloy

ORIGINAL RESEARCH PAPER McNeela, E. A. et al. Pneumolysin activates the NLRP3 inflammasome and promotes proinflammatory cytokines independently of TLR4. PLoS Pathog. 6 , e1001191 (2010)

FURTHER READING Kadioglu, A. et al. The role of Streptococcus pneumoniae virulence factors in host respiratory colonization and disease. Nature Rev. Microbiol. 6, 288-301 (2008) 